\title{
The outcome of root canal treatment. A retrospective study within the armed forces (Royal Air Force)
}

\author{
J. D. Peak, ' S. J. Hayes, ${ }^{2}$ S. T. Bryant, ${ }^{3}$ P. M. H. Dummer, ${ }^{4}$
}

\begin{abstract}
Objective The objective of this study was to investigate the outcome of conventional root canal treatment in a general practice setting within the Royal Air Force dental service.

Design Retrospective review.

Methods Teeth that had been root-filled for 12 months or more by Royal Air Force dental practitioners in patients attending a large Royal Air Force dental centre were included in the study. Following clinical and radiographic review the root fillings were classified as 'definitely successful', 'probably successful' or 'failed'. The effect on success of several variables on the outcome was investigated.

Results Out of a total of 406 teeth, 59\% were maxillary teeth and $41 \%$ were mandibular teeth. Sixty-nine per cent of the total sample had pre-existing periapical radiolucencies. Cold lateral condensation of gutta-percha was the most widely used filling technique ( $64 \%$ of all cases). Fifty per cent of the teeth had root fillings within $2 \mathrm{~mm}$ of the radiographic apex, $32 \%$ were greater than $2 \mathrm{~mm}$ from the radiographic apex and $18 \%$ were overfilled. Cold lateral condensation was the most successful (92\% overall) filling technique. Maxillary anterior teeth had a better success rate $(96 \%)$ than other tooth types. Teeth with pre-existing periapical radiolucencies had a higher success rate $(87 \%)$ than those cases where there was no pre-existing periapical radiolucency (80\%). Root fillings that were less than $2 \mathrm{~mm}$ from the radiographic apex of the tooth had a higher success rate $(88 \%$ overall) than those that were greater than $2 \mathrm{~mm}$ from the radiographic apex (77\% overall). Of the 406 cases, $57 \%(n=231)$ were classified as definitely successful, $28 \%(n=114)$ were classified as probably successful and $15 \%(n=62)$ were classified as failures. Thus, the overall success rate combining definitely successful and probably successful root fillings was $85 \%(n=344)$. Conclusions Root fillings placed using cold lateral condensation of gutta-percha to within $2 \mathrm{~mm}$ of the radiographic apex of the tooth were associated with the best outcome.
\end{abstract}

\footnotetext{
Guccess rates of treatment modalities are an important part of Sevidence-based practice; they are the basis of treatment planning and prognostic considerations. A successful outcome for root canal treatment relies on adequate removal of micro-organisms from the canal system and prevention of recolonisation or propagation of residual micro-organisms through the placement of a root filling that obturates the space entirely, combined with a restoration that produces a satisfactory coronal seal. ${ }^{1}$ Despite being

${ }^{1 *}$ Clinical Research Fellow, ${ }^{2}$ Lecturer, ${ }^{3}$ Nonclinical Research Fellow, ${ }^{4}$ Professor, Department of Adult Dental Health, University of Wales College of Medicine, Heath Park, Cardiff, CF14 4XY, UK

${ }^{*}$ Correspondence to: $S$. J. Hayes

REFEREED PAPER

Received 27.09.99; Accepted 11.09.00

(C) British Dental Journal 2001; 190: 140-144
}

one of the most technically demanding procedures in restorative dentistry, ${ }^{2}$ conventional root canal treatment completed in dental schools or by specialists has been shown to be highly successful. ${ }^{3-7}$ Molven and Halse ${ }^{6}$ examined root canal treatment performed by dental students and found success rates of $68 \%$ for teeth with preexisting periapical radiolucencies, and $91 \%$ in teeth without preexisting periapical radiolucencies. Heling and Tamshe ${ }^{4}$ reported an overall success rate of $70 \%$ in 213 teeth that were also treated by dental undergraduates. Smith et al. ${ }^{7}$ reported an overall success rate of $84 \%$ in 821 teeth that had root fillings placed by postgraduate students and staff in a dental hospital. There are, however, few studies of the outcome of conventional root canal treatment performed by general dental practitioners. Barbakow et al. ${ }^{8,9}$ evaluated root canal treatment performed in general practice in patients aged 10 to 80 years over a nine year period. Out of a total of 566 teeth, almost one quarter were maxillary incisors and one fifth were maxillary premolars; the majority of teeth were root-filled with gutta-percha and sealer. Teeth that were root-filled to the radiographic apex of the tooth were more successful than teeth that were filled short of the apex; the overall success rate was $87 \%$.

Inferior technical quality of root fillings is considered to be the main cause of clinical failure. ${ }^{10,11}$ Incomplete obturation of the root canal leaves residual space for microbial colonisation and proliferation and may also imply that cleaning was incomplete. Grieve and McAndrew $^{12}$ reported that the majority of root fillings in their study of teeth with post retained crowns were unsatisfactory. Saunders et al. ${ }^{13}$ found that $39 \%$ of root fillings were greater than $2 \mathrm{~mm}$ from the radiographic apex and stressed the need to improve quality of root canal treatment in general dental practice. They confirmed that root fillings judged to be adequate radiographically were associated with a reduced incidence of periapical radiolucency. In a radiographic assessment of root fillings performed in general dental practice Dummer ${ }^{14}$ showed that only $10 \%$ of cases fulfilled technical criteria for standards of care as defined by the European Society of Endodontology. 15

The aim of this retrospective study was to investigate the outcome of conventional root canal treatment in an armed forces (Royal Air Force) practice when assessed using defined clinical and radiographic criteria. This study is a continuation of a preliminary investigation; ${ }^{16}$ the present report includes substantially more teeth.

\section{Methods}

Selection of cases

This study includes teeth that had been root filled by a large number of Royal Air Force dental practitioners in patients attending a large Royal Air Force dental centre. The teeth were reviewed clinically using defined criteria for success / failure, either by the principle author (JDP) or by 1 of 3 other clinicians. The radiographs were reviewed by 1 clinician (JDP). From this sample only teeth that had been root filled for 12 months or greater were selected for inclusion. 
Table I Percentage success rate and actual numbers () in terms of filling technique by tooth type

\begin{tabular}{|c|c|c|c|c|c|c|c|c|}
\hline & \multicolumn{2}{|c|}{ Anterior } & \multicolumn{2}{|c|}{ Premolar } & \multicolumn{2}{|c|}{ Molar } & \multicolumn{2}{|c|}{ Overall } \\
\hline & Max & Mand & $\operatorname{Max}$ & Mand & Max & Mand & Success & Failure \\
\hline Lateral condensation & $96(67)$ & $90(26)$ & $91(39)$ & $93(25)$ & $91(30)$ & $89(50)$ & $92(237)$ & $8(2 I)$ \\
\hline Single cone gutta-percha & $63(26)$ & $90(9)$ & $70(9)$ & $90(9)$ & $67(4)$ & $20(I)$ & $68(58)$ & $32(27)$ \\
\hline Silver/titanium point & $94(15)$ & $100(2)$ & $25(I)$ & $100(2)$ & $37(3)$ & $75(9)$ & $73(32)$ & $27(44)$ \\
\hline Endomethasone & $100(2)$ & $100(8)$ & $100(3)$ & $100(2)$ & $100(2)$ & $0(0)$ & $89(17)$ & II (2) \\
\hline Overall success & $85(110)$ & $92(45)$ & $83(52)$ & $93(38)$ & $80(39)$ & $80(60)$ & & \\
\hline Overall failures & $15(19)$ & $8(4)$ & $17(11)$ & $7(3)$ & $20(10)$ & $20(15)$ & & \\
\hline
\end{tabular}

In cases where a tooth had been extracted after a root filling had been placed, the reason for extraction was ascertained from the clinical records. If the extraction was related to failure of the root canal treatment the tooth was included in the study; if not then it was excluded. Teeth in which different canals were filled with different filling materials were also excluded. This produced a final sample of 406 cases.

\section{Assessment of root fillings}

The following variables were recorded for each case by one of the authors (JDP):

Material type The filling material and obturation technique were recorded for each tooth; this was either lateral condensation of gutta-percha, single cone gutta-percha, silver/titanium point technique or Endomethasone (Septodont, St. Maur, France) alone.

Tooth type This was categorised as maxillary anterior, maxillary premolar, maxillary molar, mandibular anterior, mandibular premolar or mandibular molar.

Presence or absence of an initial (pretreatment) periapical radiolucency This was assessed by one examiner using a $4 \mathrm{x}$ hand lens under even illumination and with extraneous light excluded. Pathology was deemed to exist if there was widening of the periapical periodontal ligament space or there was bone loss on the pretreatment radiograph. Radiographs were taken in various clinics with differing X-ray and processing equipment; use of paralleling devices was limited.

Distance of root filling from the radiographic apex This was measured directly from the post-obturation radiograph and categorised according to whether the root filling was less than $2 \mathrm{~mm}$ from the radiographic apex, $2 \mathrm{~mm}$ or greater from the radiographic apex or overfilled. In multi-rooted teeth in which all canals were not filled to the same length and where there was no apical area the average distance of the root fillings from the radiographic apices of that tooth was recorded. In multi-rooted teeth where only one root was associated with an area, that root was selected for assessment.

The length of time elapsed since placement of the root filling This was grouped as either less than 3 years, or 3 years and greater.

The outcome of treatment was classified as:

Table 2 Success rate (\%) in terms of filling technique with or without an initial apical radioluceny

\begin{tabular}{lccc}
\hline & No initial radiolucency & Initial radiolucency & All \\
\hline Lateral condensation & 92 & 92 & 92 \\
Single-cone gutta-percha & 54 & 75 & 68 \\
Silver/titanium & 60 & 79 & 73 \\
Endomethasone & 100 & 83 & 89 \\
All & 80 & 87 & \\
\hline
\end{tabular}

- Definite success If there were no clinical signs or symptoms of pathology, i.e. no symptoms reported by the patient, no buccal erythema, swelling, discharging sinus, tooth mobility nor tenderness to gentle percussion with a mirror handle and no radiographic evidence of pathology.

- Probable success If there were no clinical signs or symptoms of pathology and radiographic evidence of a reduced periapical area since placement of the root filling.

- Failure If there were clinical signs of pathology and/or bone loss equal to or greater than the original periapical radiolucency.

All radiographs were interpreted by one examiner. The cut-off point for success or failure was taken as being that of the most recent available radiograph, unless previous clinical signs were suggestive of earlier failure in which case the earlier date was chosen and recorded.

Examiner reliability was assessed by re-examining 60 sets of preand post-treatment radiographs on a separate occasion without knowledge of the first readings. Intra-examiner consistency was measured using Cohen's unweighted kappa statistic. ${ }^{17}$

\section{Data management}

The data was recorded on paper and then encoded and transferred to computer. A software package (Microsoft Excel) was used to assess the data descriptively.

\section{Results}

\section{General findings}

Of the 406 teeth included in the study, 64\% ( $n=258)$ were filled by lateral condensation of gutta-percha, $21 \%(\mathrm{n}=85)$ were filled using a single cone gutta-percha technique, $11 \%(n=44)$ were filled using a single silver or titanium point technique and $5 \%(n=19)$ were filled with Endomethasone (Septodont) alone.

Of the 406 teeth included in the study, 59\% ( $n=241)$ were maxillary teeth and $41 \%(n=165)$ were mandibular teeth. Thirty-two per cent $(n=129)$ were maxillary anterior teeth, $15 \%(n=63)$ were $\max$ illary premolars and $12 \%(n=49)$ were maxillary molars. Twelve per cent $(n=49)$ were mandibular anterior teeth, $10 \%(n=41)$ were mandibular premolars and $18 \%(n=75)$ were mandibular molars.

Sixty-nine per cent $(n=280)$ of the total sample had an initial periapical radiolucency.

Table 3 Success rate (\%) in terms of filling technique by distance of root filling from radiographic apex $(\mathrm{mm})$.

Distance from radiographic apex $(\mathrm{mm})$

\begin{tabular}{lccc} 
& Overfilled & $<2$ & $\geq 2$ \\
\hline Lateral condensation & 93 & 93 & 88 \\
Single cone gutta-percha & 60 & 78 & 57 \\
Silver/titanium point & 92 & 92 & 62 \\
Endomethasone & 100 & 60 & 100 \\
All & 89 & 88 & 76 \\
\hline
\end{tabular}


Fifty per cent $(\mathrm{n}=205)$ of root fillings were within $2 \mathrm{~mm}$ of the radiographic apex, $32 \%(n=129)$ were $2 \mathrm{~mm}$ or greater from the radiographic apex and $18 \%(n=72)$ were overfilled.

Of the total, $57 \%(n=231)$ were classified as definitely successful, $28 \%(n=114)$ were classified as probably successful and $15 \%(n=62)$ were classified as failures. Thus, the overall success rate combining definitely successful and probably successful root fillings was $85 \%$ $(\mathrm{n}=344)$.

The percentage success rates in terms of filling material and tooth type are shown in Table 1 . There were only 2 failures associated with Endomethasone (Septodont) and both these were in mandibular molars. The highest success rate (96\%) for all techniques other than Endomethasone (Septodont) alone was seen in maxillary anterior teeth filled with lateral condensation. Single cone techniques using either gutta-percha, silver or titanium produced inconsistent results throughout all tooth types.

The success rates in terms of filling technique and whether or not there were pre-existing periapical radiolucencies are detailed in Table 2. The trend was for higher success rates in those cases where a periapical radiolucency was present before treatment (87\% versus 80\%). Root fillings of Endomethasone (Septodont) alone, achieved higher success rates in teeth where there was no periapical radiolucency before treatment (100\% versus $83 \%$ ).

The percentage success rates in terms of filling technique and distance of root filling from the radiographic apex are detailed in Table 3. With the exception of Endomethasone (Septodont) alone, fillings placed to within $2 \mathrm{~mm}$ of the radiographic apex were associated with higher success rates than fillings that were $2 \mathrm{~mm}$ or greater from the radiographic apex. Overall, teeth that were overfilled had higher success rates than those that were $2 \mathrm{~mm}$ or more from the radiographic apex.

The success rates in terms of filling technique by follow-up period are shown in Table 4. Overall, single cone techniques using either gutta-percha, silver or titanium had substantially lower success rates when reviewed within the first 3 years compared with lateral condensation or Endomethasone (Septodont). Those teeth reviewed 3 years or more after placement of a root filling had a higher success rate, largely as a result of the enhanced outcome in the single cone techniques.

Table 4 Success rate (\%) of filling techniques by follow-up period

\begin{tabular}{lccc}
\hline & $<3$ years & $\geq 3$ years & All \\
\hline Lateral condensation & 91 & 93 & 92 \\
Single-cone gutta-percha & 30 & 80 & 68 \\
Silver/titanium & 25 & 77 & 73 \\
Endomethasone & 100 & 87 & 89 \\
All & 82 & 86 & 85 \\
\hline
\end{tabular}

Table 5 shows the success rates in terms of tooth type and whether or not there was a pre-existing periapical radiolucency. The highest success rates were seen in teeth with pre-existing periapical radiolucencies and in mandibular anteriors and premolars; most failures occurred in molar teeth. Table 6 details the success rates in terms of tooth type and distance of the root filling from the radiographic apex. Other than maxillary anteriors and mandibular molars, the success rates were highest in teeth that were overfilled. Maxillary anterior and mandibular molar teeth that were overfilled were associated with failure rates of $23 \%$ and $25 \%$ respectively. With the exception of maxillary premolars and mandibular molars, teeth that were root filled to within $2 \mathrm{~mm}$ of the radiographic apex had high success rates. Table 7 details the success rates in terms of tooth type by follow-up period. Mandibular anteriors and premolars that were reviewed within 3 years of placement of a root filling had higher success rates than other tooth types. Maxillary anterior teeth that were reviewed within 3 years of placement of a root filling had lower suc-
Table 5 Success rate (\%) and actual numbers ( ) in terms of tooth type with or without an initial apical radiolucency

\begin{tabular}{ccccc} 
Anterior & Premolar & Molar & All \\
Max Mand & Max Mand & Max Mand & \\
\hline
\end{tabular}

No pre-existing

radiolucency $76(26) \quad 100(7) \quad 83(19) \quad 89(16) \quad 75(18) \quad 75(15) \quad 80(101)$

Pre-existing

radiolucency $88(84) \quad 90(38) \quad 82(33) \quad 96(22) \quad 84(19) \quad 82(45) \quad 87(243)$

cess rates. In cases that were reviewed 3 years or more after completion of root canal treatment the trend was for success rates in posterior teeth to be lower than anterior teeth. Overall, the cases that were reviewed within 3 years of root canal treatment had a lower success rate than those cases that were reviewed 3 years or more after root canal treatment. Table 8 outlines the success rates in terms of tooth type, whether there was pre-existing periapical radiolucency or not and the distance of the root filling from the radiographic apex. In general, teeth that did not have pre-existing periapical radiolucencies and were overfilled were associated with low success rates. Conversely, teeth that did have pre-existing periapical radiolucencies and were overfilled had higher success rates.

Table 6 Success rate (\%) and actual numbers () in terms of tooth type and distance of root filling from the radiographic apex ( $\mathrm{mm})$

\begin{tabular}{lrrrrrrrr}
\hline & \multicolumn{2}{c}{ Anterior } & \multicolumn{2}{c}{ Premolar } & \multicolumn{2}{c}{ Molar } & \multirow{2}{*}{ All } \\
& Max & Mand & Max & Mand & Max & Mand & \\
\hline Overfilled & $77(20)$ & $100(8)$ & $100(11)$ & $100(6)$ & $100(4)$ & $75(15)$ & $89(64)$ \\
$<2 \mathrm{~mm}$ & $93(71)$ & $94(31)$ & $74(26)$ & $95(19)$ & $74(14)$ & $100(20)$ & $88(18 I)$ \\
$\geq 2 \mathrm{~mm}$ & $76(19)$ & $75(6)$ & $88(15)$ & $87(13)$ & $81(2 I)$ & $66(25)$ & $77(99)$
\end{tabular}

Table 9 shows the differences in success rate in terms of the distance of the root filling from the radiographic apex of the tooth by follow-up period. Teeth that were root filled to within $2 \mathrm{~mm}$ of the radiographic apex and that were reviewed within 3 years of root canal treatment had a higher success rate than those cases that had root fillings that were greater than $2 \mathrm{~mm}$ from the radiographic apex or overfilled. In cases that were reviewed 3 years or more after root canal treatment, teeth that were overfilled or had root fillings within $2 \mathrm{~mm}$ of the apex had a higher success rate than those where the root filling was greater than $2 \mathrm{~mm}$ from the apex.

Table 7 Success rates (\%) and actual numbers ( ) in terms of tooth type over time

\begin{tabular}{ccccccc}
\hline \multicolumn{2}{c}{ Anterior } & \multicolumn{2}{c}{ Premolar } & \multicolumn{2}{c}{ Molar } & \multirow{2}{*}{ All } \\
Max & Mand & Max & Mand & Max & Mand & \\
\hline$<3$ years 73(33) $100(13)$ & $77(24)$ & $100(19)$ & $83(19)$ & $82(31)$ & $82(139)$ \\
$\geq 3$ years 92(77) & $89(29)$ & $87(28)$ & $86(19)$ & $77(20)$ & $78(29)$ & $86(205)$ \\
\hline
\end{tabular}

\section{Discussion}

It is important to emphasise that the cohort studied in this survey may not be representative of those patients receiving a root filling in the United Kingdom as a whole. As a result it was not considered appropriate to analyse the data statistically. However, despite this potential shortcoming the information is both interesting and valuable.

Royal Air Force personnel are subject to age restrictions and so the extremes of the normal population will not be present. Dental surgeons in the Royal Air Force are salaried and may not be comparable to dentists working in Health Service practice who are mainly paid a fee per item of work. Further, the radiographs used in the study were not taken in standardised manner; the inevitable 
Table 8 Success rates (\%) and actual numbers ( ) in terms of tooth type, distance from the radiographic apex and with or without an initial apical radiolucency

\begin{tabular}{ccccc}
\hline Anterior & Premolar & Molar & All \\
Max Mand & Max & Mand & Max Mand & \\
\hline
\end{tabular}

\section{Overfilled -}

initial radiolucency $8 \mathrm{I}(\mathrm{I7}) \quad 100(6) 100(10) \quad 100(4) 100(4) 100(\mathrm{II}) \quad 93(52)$

\section{Overfilled -}

no radiolucency $60(3) 100(2) \quad 100(1) \quad 100(2) \quad * \quad 67(4) \quad 75(12)$

$<2 \mathrm{~mm}-$

initial radiolucency 9I (58) $93(26) \quad 68(15)$ I00(I2) $86(6)$ I00(I6) 89 (I33)

$<2 \mathrm{~mm}-$

no radiolucency $93(13) \quad 100(5) \quad 85(\mathrm{II}) \quad 87(7) \quad 67(8) \quad 100(4) \quad 86(48)$

$\geq 2 \mathrm{~mm}-$

initial radiolucency $90(9) \quad 75(6) \quad 100(8) \quad 86(6) \quad 79(11) \quad 64(18) \quad 77(58)$

$\geq 2 \mathrm{~mm}-$

no radiolucency $\quad 67(10) \quad * \quad 78(7) \quad 87(7) 83(10) \quad 70(7) \quad 76(4 I)$

$*$ no data

changes in beam / film angulation between pre- and post-operative views will have affected the images of teeth and bone. ${ }^{18}$ In addition, the radiographs were examined by one operator only; other studies have suggested that more than one examiner is more appropriate. ${ }^{7}$ Intra-examiner variability was examined using the Cohen's unweighted kappa formula and was found to be within acceptable limits ( $=0.61$ for diagnosis and $=0.71$ for distance indicating substantial agreement). ${ }^{16}$ Controlled prospective study of the many aetiological factors contributing to the success or failure of root canal treatment would be the method of choice to determine outcomes, however, such studies present significant clinical, research and statistical challenges and are rarely reported.

Cold lateral condensation of gutta-percha in conjunction with a root canal sealer is the most widely accepted technique for obturating root canals ${ }^{19,20}$ and was the most widely used filling technique in the present study (Table 1). The high prevalence of the lateral condensation technique in this study contrasts with the findings of studies of root canal treatment performed in general practice $21-23$ that showed a much greater use of single cone gutta-percha and paste-only techniques. Presumably, this simply reflects the different practice profile of RAF dentists.

Cold lateral condensation is a simple and versatile technique that has consistently been shown to produce good results; it was the most successful (92\% success) filling technique in this study (Table 1). Lateral condensation of gutta-percha showed high success rates with all tooth types. The high success rate (89\%) of Endomethasone (Septodont) in the present work is comparable with that of Boggia ${ }^{23}$ who reported no failures. Boggia was a Royal Air Force practitioner and it is likely that many of the cases filled with Endomethasone (Septodont) in this study were part of the sample he reported. However, only a small number of teeth $(n=19)$ were filled with Endomethasone (Septodont) alone, the majority being anteriors (10 out of 19), perhaps reflecting concerns over its use. ${ }^{15,24,25}$ Single gutta-percha cone or silver/titanium point techniques had lower success rates ( $68 \%$ and $73 \%$ respectively). The reduced success rates noted with single cone techniques suggests that the minimally flared preparations required for such filling techniques do not result in adequate cleaning, either mechanically or chemically, and that the filling technique provides a less effective seal.

Maxillary anterior teeth had better success rates than other types of teeth (Table 1). Access is better at the front of the mouth and the root canal anatomy is usually more amenable to treatment, i.e. single, straight and more circular allowing better cleaning and simpler obturation. Multi-rooted teeth with curved root canals present more of a challenge in all stages of root canal treatment and their
Table 9 Success rate (\%) and actual numbers () in terms of the distance of the root filling from the radiographic apex $(\mathrm{mm})$ by followup period

\begin{tabular}{lccr}
\hline & \multicolumn{3}{c}{ Distance from radiographic apex $(\mathrm{mm})$} \\
& Overfilled & $<2$ & $\geq 2$ \\
\hline$<3$ years & $82(23)$ & $86(7 \mathrm{I})$ & $78(46)$ \\
$\geq 3$ years & $93(4 \mathrm{I})$ & $90(110)$ & $76(53)$ \\
\hline
\end{tabular}

higher failure rates may be due to factors other than filling technique alone, e.g. lack of access, poorer cleaning, accessory anatomy.

Almost a third of the sample were root filled without pre-existing periapical radiolucency, i.e. on clinical grounds alone (Tables 2, 5 and 8). Generally, teeth with periapical radiolucencies had better success rates $(87 \%)$ than those without $(80 \%)$, which is in contrast to most studies that have investigated this factor. ${ }^{6,7}$ This might be explained by the high proportion $(69 \%)$ of teeth with pre-existing periapical radiolucencies in the present study. Interestingly, the presence of periapical radiolucency had no effect on the success rate of teeth filled with laterally condensed gutta-percha ( $92 \%$ in both cases). The lack of standardisation of radiographic technique may also have influenced the results.

The technical quality of root canal treatment was assessed indirectly by measuring the distance of the root filling from the radiographic apex of the tooth (Table 3, 6 and 8). Sjögren et al. ${ }^{26}$ showed that canals filled to within $2 \mathrm{~mm}$ of the radiographic apex were associated with higher success rates; that is, when the whole root canal system had been cleaned to eliminate microorganisms, and shaped to facilitate three-dimensional obturation in order to inhibit reinfection. Not surprisingly, and in agreement with Sjögren et al. ${ }^{26}$ and others, ${ }^{9,13}$ some of the lowest failure rates were noted in teeth that had root fillings within $2 \mathrm{~mm}$ of the radiographic apex. This probably implies that the canals were also cleaned and shaped more effectively because of the enhanced canal flare required as well as being filled more completely. Lateral condensation had a higher success rate than the other techniques in cases where the root filling was more than $2 \mathrm{~mm}$ from the radiographic apex. Presumably the same explanation would apply for this group.

Almost one fifth of the teeth in the present study were overfilled. Where pre-existing periapical radiolucency was present overfilling seemed to be of some benefit (Table 8). In the absence of a pre-existing periapical radiolucency overfilling resulted in a lower success rate (Table 7). The high success rate in the present work of teeth that were overfilled contrasts with the findings of some other studies. ${ }^{5-7}$ Interestingly, however, overfilling did not produce the worst results in the work of Barbakow et al., ${ }^{8-9}$ and Boggia ${ }^{23}$ deliberately overfilled 52 non-vital teeth with Endomethasone (Septodont) containing paraformaldehyde with no failures. Again, the non-standardised method of obtaining the radiographic images may have affected the outcome.

Lateral condensation had consistently high success rates in cases that were reviewed within 3 years of root canal treatment and in those reviewed after this period (Table 4). Single-cone gutta-percha and silver/titanium point techniques had low success rates in the group reviewed within the first 3 years after placement $30 \%$ and $25 \%$ success respectively). In cases that were reviewed 3 years or more after root canal treatment the success rates for the same techniques were higher ( $80 \%$ and $77 \%$ success respectively), but not as high as lateral condensation (93\% success). There were no failed root-fillings in any of the mandibular anterior and premolar teeth reviewed within 3 years of placement (Table 7). In this study, overfilled teeth had the highest success rate $(93 \%)$ in cases that were reviewed 3 years or more after root canal treatment (Table 9).

The overall success rate of root fillings in the present study was $85 \%$ and compares well with studies of root canal treatment performed in dental schools and hospitals. ${ }^{6,7}$ Inadequate funding has 
been implicated as a barrier to improving endodontic care in Health Service general practice 27 and a higher standard of work might be expected when clinicians are not directly concerned with financial constraints; this appears to be the case in the present study. For example, $50 \%$ of root fillings were within $2 \mathrm{~mm}$ of the radiographic apex compared to $39 \%$ reported by Saunders et al. ${ }^{13}$ and $39 \%$ reported by Dummer. ${ }^{14}$ Since technical quality of root canal treatment has a major impact on the outcome it is critical that the quality of the original treatment complies with standards of care advocated by internationally accepted bodies. ${ }^{15,28}$ However, the authors accept that the sample studied was selective, as was the group of clinicians performing the work. These factors, and the potential problems with the non-standardised radiographs should caution against direct comparison of results with other studies.

\section{Conclusions}

Within the limitation of the study root fillings using lateral condensation of gutta-percha had higher success rates than other techniques. Maxillary anterior teeth had higher success rates than other tooth types. Root fillings that were within $2 \mathrm{~mm}$ of the radiographic apex were more successful than root fillings that were $2 \mathrm{~mm}$ or greater from the apex or overfilled. Single cone gutta-percha and silver/titanium point techniques were associated with a high failure rate in cases that were reviewed within 3 years of root canal treatment.

Overall, the highest success rates were seen in teeth filled with laterally condensed gutta-percha to within $2 \mathrm{~mm}$ of the radiographic apex of the tooth.

The authors wish to thank the Chief Executive, Defence Dental Agency for his permission to publish this paper and Dr $R$ Walker for helping with the original design of the study. The authors also wish to acknowledge the help given by RAF Dental Officers Gp Capt E Donnison, Sqn Ldr J McDavitt and Sqn Ldr E Rhodes who facilitated data collection and that given by the support staff from RAF St Athan who provided administrative assistance.

1 Briggs P F A, Scott B J J. Evidence-based dentistry: endodontic failure how should it be managed? Br Dent J 1997; 183: 159-64.

2 Brookman D J. Vocational trainees' views of their undergraduate endodontic training and their vocational training experience. Int Endod J 1991; 24: 178-86.

3 Grossman L I, Shepard L I, Pearson L A. Roentgenologic and clinical evaluation of endodontically treated teeth. Oral Surg Oral Med Oral Path $1964 ; 17368-74$

4 Heling B, Tamshe A. Evaluation of the success of endodontically treated teeth. Oral Surg 1970; 30: 533-6.

5 Swartz D B, Skidmore A E, Griffin J A. Twenty years of endodontic success and failure. J Endod 1983; 9: 198-202.

6 Molven O, Halse A. Success rates for gutta-percha and Kloroperka N-O root fillings made by undergraduate students: radiographic findings after 10-17 years. Int Endod J 1988; 21: 243-50.
7 Smith C S, Setchell D, Harty F J. Factors influencing the success of conventional root canal therapy - a five-year retrospective study. Int Endod J 1993; 26: 321-33.

8 Barbakow F H, Cleaton-Jones P E, Friedman D. An evaluation of 566 cases of root canal therapy in general dental practice. 1. Diagnostic criteria and treatment details. J Endod 1980; 6: 456-60.

9 Barbakow F H, Cleaton-Jones P E, Friedman D. An evaluation of 566 cases of root canal therapy in general dental practice. 2. Postoperative observations. J Endod 1980; 6: 485-9.

10 Eckerbom M, Andersson J-E, Magnusson T. A longitudinal study of changes in frequency and technical standard of endodontic treatment in a Swedish population. Endod Dent Traumatol 1989; 5: 27-31.

11 DeCleen M J H, Schuurs A H B, Wesselink P R, Wu M-K. Periapical status and prevalence of endodontic treatment in an adult Dutch population. Int Endod J 1993; 26: 112-9.

12 Grieve A R, McAndrew R. A radiographic study of post-retained crowns in patients attending a dental hospital. Br Dent J 1993; 174: 197-201.

13 Saunders W P, Saunders E M, Sadiq J, Cruickshank E. Technical standard of root canal treatment in an adult Scottish sub-population. Br Dent J 1997; 182: 382-6.

14 Dummer P M H. The quality of root canal treatment provided by general dental practitioners working within the general dental services of England and Wales. Part 2. Dental Profile (J Dent Pract Board Eng Wales) 1998; 19: 8-10.

15 Consensus report of the European Society of Endodontology on quality guidelines for endodontic treatment. Int Endod J 1994; 27: 115-24.

16 Peak J D. The success of endodontic treatment in general dental practice: A retrospective clinical and radiographic study. Primary Dental Care 1994; 1: 9-13.

17 Bulman J S, Osborn J F. Measuring diagnostic consistency. Br Dent J 1989; 166: 377-81.

18 Fava L R G, Dummer P M H. Periapical radiographic techniques during endodontic diagnosis and treatment. Int Endod J 1997; 4: 250-61.

19 Dummer P M H. Comparison of undergraduate endodontic teaching programmes in the United Kingdom and in some dental schools in Europe and the United States. Int Endod J 1991; 24: 169-77.

20 Qualtrough A J E, Dummer P M H. Undergraduate endodontic teaching in the United Kingdom: an update. Int Endod J 1997; 30: 234-40.

21 Report to the Council of the British Endodontic Society. The practice of endodontics by different groups of dentists in England. Int Endod J 1983; 16: 185-91.

22 Dummer P M H. The quality of root canal treatment in the general dental services. Dental Profile (J Dent Pract Board Eng Wales) 1997;17: 5-6.

23 Boggia R. A single-visit treatment of septic root canals using periapically extruded Endomethasone. Br Dent J 1983; 155: 300-5.

24. Fanibunda K B. Adverse response to endodontic material containing paraformaldehyde. Br Dent J 1984; 157: 231-5.

25. Erison R, Yucel T, Kucukay S. Endomethasone root canal filling material in the mandibular canal: a case report. Oral Surg Oral Med Oral Path 1993; 63 343-5.

26. Sjögren U, Hagglund B, Sundqvist G, Wing K. Factors affecting the longterm results of endodontic treatment. J Endod 1990; 166: 498-504.

27. McColl E, Smith M, Whitworth J, Seccombe G, Steele J. Barriers to improving endodontic care: the views of NHS practitioners. Br Dent J 1999 186: $564-8$.

28. Quality Assurance Guidelines. Chicago, American Association of Endodontists, 1987; 1-27. 